\section{Análisis del concepto de serenidad en relación con el apoyo psicológico y emocional del paciente crónico}

\section{Analysis of the concept of serenity in the emotional support of the chronic patient}

Geòrgia Papiol Espinosa ${ }^{1}$

Margaretha Norell Pejne ${ }^{2}$

Mercedes Abades Porcel ${ }^{3}$

1. Enfermera asistencial. Unidad de Psicogeriatría. Complejo Asistencial en Salud Mental Benito Menni. Sant Boi de Llobregat. Barcelona. España.

2. Profesora de la Escuela de Enfermería. Academia de Salud y Bienestar. Universidad de Halmstad. Suecia.

3. Profesora de la Escuela Universitaria de Enfermería EUI-Sant Pau. Centro adscrito a la Universitat Autònoma de Barcelona (UAB). Barcelona. España.

*Autor para correspondencia.

Correo electrónico: georgiape@hotmail.com (Geòrgia Papiol Espinosa).

\section{RESUMEN}

El proceso de envejecimiento y las enfermedades crónicas implican cambios vitales en el adulto mayor que suponen, en muchas ocasiones, acontecimientos estresantes que derivan en un malestar psicológico y un deterioro de la calidad de vida. La vejez es una etapa caracterizada por sentimientos de pérdidas y disminución de capacidades funcionales y afectivas. Se investiga la serenidad como estrategia de afrontamiento en relación con el apoyo psicológico y emocional para mejorar las capacidades del adulto mayor para afrontar los problemas o dificultades que conllevan las consecuencias del envejecimiento. Objetivo: Analizar el concepto de serenidad en relación con el apoyo psicológico y emocional

del paciente crónico. Metodología: Revisión de la bibliografía. La

búsqueda se realizó en PubMed, CINAHL, Cochrane Library y Medline. Se acotó al período 1999-2017, incluyendo aquellos estudios que solo aplican el concepto de serenidad para la promoción de la salud mental. Resultados: De los 40 estudios revisados, 8 hacían referencia al envejecimiento y la cronicidad; 15 a trastornos afectivos en la cronicidad;

13 al apoyo emocional y psicológico en el paciente mayor crónico,

y 4 a la serenidad y cuidado enfermero. Posteriormente al análisis se identificaron tres temas relacionados: apoyo emocional en la cronicidad, concepto de serenidad y bienestar psíquico y emocional, definiendo atributos y niveles de serenidad, apoyo emocional en la cronicidad.

Conclusiones: El concepto de serenidad puede representar una herramienta que fomente el bienestar psíquico y emocional del paciente mayor crónico, ayudando a aceptar y manejar la situación de salud. Resulta necesaria una mayor evidencia científica sobre el concepto y su uso en los profesionales enfermeros.

PALABRAS CLAVE: Cronicidad, envejecimiento, apoyo psicológico y emocional, serenidad, cuidados enfermeros.
The aging process and chronic diseases involve vital changes in the elderly that, in many cases, involve stressful events that lead to psychological discomfort and a deterioration in the quality of life. Old age is a stage characterized by feelings of loss and decreased functional and affective abilities. Serenity is investigated as a coping strategy in relation to psychological and emotional support to improve the abilities of the elderly to face the problems or difficulties that entail the consequences of aging. Objective: To analyze the concept of serenity in relation to the psychological and emotional support of the chronic patient. Methodology: Review of the literature. The search was performed in PubMed, CINAHL, Cochrane Library, Medline. The 1999-2017 period was included, including those studies that only apply the concept of serenity for the promotion of mental health. Results: Of the 40 studies reviewed, 8 referred to aging and chronicity; 15 to affective disorders in the chronicity; 13 to emotional and psychological support in the chronically elderly patient; 4 to serenity and nursing care. Three related topics were identified: emotional support in chronicity, the concept of serenity and psychic and emotional well-being, defining attributes and levels of serenity, emotional support in chronicity. Conclusions: The serenity can represent a tool that promotes the psychic and emotional well-being of the chronic elderly patient, helping to accept and manage the health situation. Greater scientific evidence on the concept and its use in nursing professionals is necessary.

KEYWORDS: chronicity, aging, psychological and emotional support, serenity, nursing care.

\section{- INTRODUCCIÓN}

España, a mediados del siglo xxi, será uno de los países más envejecidos, con un incremento mayor de personas con problemas de salud crónicos en situación de plurimorbilidad ${ }^{1}$. El proceso de envejecimiento y las enfermedades crónicas implican cambios vitales en el adulto mayor, que requieren de un importante esfuerzo de adaptación y limitación en la evolución afectiva del adulto mayor ${ }^{2,3}$. En la vejez, la presencia de los cambios biológicos y la disminución de capacidades funcionales conllevan la aparición de patologías crónicas que, en muchas ocasiones, derivan hacia la incapacidad y la dependencia ${ }^{1}$. Estos factores pueden provocar, especialmente en el adulto mayor, altos niveles de estrés asociados a una percepción negativa de la propia salud ${ }^{4}$, siendo una de las principales causas de malestar psicológico (ansiedad, angustia, depresión) y del deterioro de la calidad de vida ${ }^{5}$. En la vejez inciden también los cambios en el estatus social, 
con la jubilación y la pérdida frecuente del rol social, y representa una etapa marcada por pérdidas de personas queridas. Todas estas circunstancias, en muchas ocasiones, provocan un retraimiento social, aislamiento, soledad, angustia y tristeza ${ }^{3,6}$.

El Sistema Nacional de Salud recomienda abordar la cronicidad como elemento indispensable que garantice una adecuada atención a los aspectos psicológicos y emocionales de las personas mayores que padecen patologías crónicas, y que permita mejorar los procesos de salud-enfermedad mediante la aceptación, adaptación, modificación de estilos de vida y adherencia al tratamiento ${ }^{7}$.

El King's Fund and the Centre for Mental Health justifica razones suficientes de dicho abordaje, ya que las personas que sufren enfermedades crónicas presentan un riesgo elevado de sufrir problemas de ansiedad y depresión; el coste de la comorbilidad resulta significativo (un 45\% más), los problemas de salud mental interactúan con los síntomas físicos del paciente, y la desatención del sufrimiento psicológico complica la sintomatología física y aumenta las visitas al médico ${ }^{8}$.

Entendiendo la salud como un estado de completo bienestar físico, mental, social y espiritual ${ }^{9}$, los profesionales de enfermería deben buscar estrategias de afrontamiento que ayuden al adulto a modular la respuesta al estrés generado por los cambios que supone el envejecimiento ${ }^{2}$. Del mismo modo, como profesionales de la salud, han de empoderar a los pacientes crónicos para reconocer y manejar sus propias necesidades psicológicas; ha de ser capaz de reconocer el malestar emocional y la presencia de trastornos psicológicos y tener las competencias para establecer y mantener relaciones de apoyo que ayuden al paciente a minimizar y controlar el dańo psicológico secundario a los procesos de cronicidad ${ }^{10}$.

Algunos estudios muestran evidencias de cómo el concepto de serenidad aporta resultados positivos en la atención a la cronicidad. Estos conceptos están reconocidos por mejorar las capacidades y potencialidades que dan al adulto mayor para afrontar los problemas o dificultades que conllevan las consecuencias del envejecimiento ${ }^{11}$.

A través del concepto de serenidad se contempla la importancia de cuidar la salud psicológica y emocional en el contexto geriátrico, entendiendo la serenidad como un estado de conciencia necesario para poder comprender y actuar en situaciones de vida desfavorables. Un estado que permita alcanzar un equilibrio físico, mental y espiritual para que la persona pueda aceptar, adaptarse y gestionar mejor las condiciones desfavorables ${ }^{12}$. No hay estudios a nivel nacional específicos sobre este tema, y planteamos nuestro interés en examinar el concepto de la serenidad como herramienta que pueda guiar las intervenciones del profesional de la salud en el apoyo emocional y psicológico del paciente crónico.

\section{- OBJETIVO}

Analizar el concepto de serenidad en relación con el apoyo emocional y psicológico del paciente crónico.

\section{- METODOLOGÍA}

Se ha realizado una revisión descriptiva de la literatura científica con la estrategia de abordaje cualitativo de Kitchenham ${ }^{13}$, desarrollada en dos etapas fundamentales. En la primera etapa se ha llevado a cabo la planificación de la investigación con sus interrogantes (tabla 1) y en la segunda etapa se ha desarrollado el protocolo de revisión (tabla 2), donde han quedado definidos los criterios de inclusión y exclusión, así como las estrategias de obtención y síntesis de datos.
Tabla 1. Planificación de la revisión

\section{Interrogantes de la investigación}

1 ¿La cronicidad en el adulto mayor implica una mayor presencia de trastornos afectivos? ¿Los profesionales enfermeros dan importancia al bienestar psíquico y emocional del paciente mayor crónico?

¿Qué significado tiene la serenidad? ¿Puede ser una herramienta

3 útil en el apoyo psicológico y emocional del paciente crónico? ¿Puede la serenidad fomentar un bienestar psíquico y emocional en el paciente crónico?

Fuente: elaboración propia.

La revisión de la bibliografía ha quedado configurada por todos los estudios consultados en las bases de datos PubMed, CINAHL, Cochrane Library y Medline. Se llevó a cabo una búsqueda de literatura nacional e internacional que cumpliera con los criterios de inclusión: artículos primarios publicados en los últimos 19 años, en relación con la atención psicológica en la cronicidad: serenidad e intervención enfermera (tabla 3). Se centró en los trabajos publicados desde 1999 hasta el 2017, seleccionando aquellos artículos que estuviesen redactados en inglés, francés y español.

La búsqueda se llevó a cabo entre noviembre de 2016 y diciembre de 2017, y se completó en abril de 2018. Se incluyeron los siguientes términos del Tesauro: "serenidad y health"/"serenity AND health", y "serenidad y cuidado"/"serenity AND caring”. Se combinaron entre ellos y con otros sinónimos empleando los operadores booleanos "AND" y "OR" para influir posibles variaciones utilizadas en la literatura. Se fueron combinando con palabras como "paciente anciano/older patient", "enfermería geriátrica/geriatric nursing", "cronicidad/chronicity", "cuidado emocional y psicológico/emotional" and (o y o AND) "psychological care".

La selección de los estudios fue llevada a cabo por dos investigadoras. A continuación, un revisor independiente analizó la relevancia de los estudios seleccionados.

Tras la búsqueda, lectura y evaluación de los estudios, se han recopilado las aportaciones de los diferentes autores, siguiendo los principales subtemas: concepto de serenidad y promoción de la salud; definición de atributos y niveles de serenidad; relación entre cronicidad y serenidad, e implicaciones en la práctica enfermera.

Una vez realizada la búsqueda y el análisis de artículos destacamos que, si bien a nivel nacional existen numerosos estudios que se centran en el abordaje del envejecimiento, la cronicidad y la dependencia de la población mayor, no se han encontrado estudios sobre el concepto principal que guía este trabajo de revisión, la serenidad. A nivel internacional destacan las aportaciones derivadas desde los Estados Unidos y de Suecia, estudios centrados en el análisis del concepto de serenidad y su interrelación con el cuidado enfermero.

El protocolo de revisión llevado a cabo se muestra en la tabla 2.

\section{- RESULTADOS}

Encontramos 23 artículos sobre envejecimiento y enfermedades crónicas y 17 sobre serenidad que cumplían los criterios de inclusión. De estos 40, hacían referencia a envejecimiento y cronicidad 8; a trastornos afectivos en la cronicidad 15; al apoyo emocional y psicológico en el paciente mayor crónico 13; y a la serenidad y cuidado enfermero 4 . 
Tabla 2. Protocolo de revisión

Criterios de inclusión

\begin{tabular}{|c|c|c|}
\hline & & Criterios de inclusión \\
\hline & 1 & Envejecimiento y cronicidad \\
\hline Se incluirán los estudios que traten sobre & 2 & Trastornos afectivos en la cronicidad \\
\hline y 2017, y que traten aspectos como: & 2 & Apoyo emocional y psicológico en el paciente mayor crónico \\
\hline & 3 & Serenidad y cuidado enfermero \\
\hline & & Criterios de exclusión \\
\hline No se incluyen los estudios que, a pesar de cont & ener & $\begin{array}{l}\text { os términos de búsqueda, no contengan información relevante } \\
\text { aspectos descritos en los criterios de inclusión }\end{array}$ \\
\hline & & Estrategia de obtención de datos \\
\hline
\end{tabular}

Fuente: elaboración propia

Del análisis de estos artículos se identificaron tres temas principales: apoyo emocional en la cronicidad, concepto de serenidad y bienestar psíquico y emocional, definición de atributos y niveles de serenidad, y apoyo emocional en la cronicidad.

El concepto de la salud ha ido evolucionando en las últimas décadas hacia un enfoque mucho más integral en el que el bienestar psicológico y emocional ha pasado a ser un componente fundamental en la percepción de la salud de las personas. Este concepto adquiere una realidad, con gran relevancia en las enfermedades crónicas. También, Gómez y Grau reflejan el impacto que tiene la cronicidad en el adulto y la incertidumbre que genera la enfermedad en la vida diaria, un distrés afectivo y emocional, consecuente de la pérdida de autonomía y de los factores culturales, ambientales y espirituales relacionados. Otros autores indican la importancia que tiene el proceso de adaptación y el saber convivir día a día con la patología crónica, los síntomas y complicaciones, los cambios físicos, psíquicos y afecciones emocionales que generan tristeza y sentimientos de culpa ${ }^{14}$, entre otros.

En esta línea, las investigaciones revisadas destacan que los pacientes con patologías crónicas tienen frecuentemente síntomas o trastornos depresivos y/o ansiedad, que influyen en el curso y gravedad de la enfermedad. Hay estudios empíricos que evidencian que hasta un $25 \%$ de los casos de pacientes que han presentado accidentes cerebrovasculares o ictus cumplen criterios de depresión mayor; en enfermedades metabólicas como la diabetes, la incidencia de depresión es tres veces superior a la de la población general; y los pacientes oncológicos presentan clínica de depresión en un $25 \%$ de los casos ${ }^{15}$. Estudios realizados en pacientes con enfermedad pulmonar obstructiva crónica constatan que el 35-40\% de los pacientes podrían presentar distintos grados de ansiedad, y un 75\%, síntomas depresivos ${ }^{16}$.

Otros autores, como Norell, Ziegert y Kihlgren, señalan que los síntomas como la ansiedad o la tristeza no deben ser vistos como un fenómeno individual, sino más bien como parte de un sistema complejo; el paciente puede expresar ansiedad como consecuencia del sufrimiento físico causado por la enfermedad, pero también puede ser una expresión de sufrimiento existencial causado por situaciones o acontecimientos de su propia vida, por lo que es importante el apoyo emocional ${ }^{17}$.

A partir de lo expuesto anteriormente queda patente la importancia y necesidad de integrar el apoyo psicológico y emocional en el abordaje asistencial a la cronicidad. A pesar de ello, la mayoría de los estudios consideran que en la atención a la cronicidad no siempre se ha dado la prioridad necesaria a los aspectos psicológicos, emocionales y sociales que influyen en la evolución de la propia enfermedad ${ }^{18}$. Autores como Norell-Pejner constatan que, en la mayoría de las ocasiones, las enfermeras no registran ninguna intervención centrada en el apoyo emocional del paciente crónico por no disponer de herramientas adecuadas en la atención comunitaria, por el desconocimiento de cómo el envejecimiento y la enfermedad afectan a las esferas psicológicas, y por el poco tiempo de que disponen para evaluar dichas necesidades ${ }^{11}$. Según datos de la OMS sobre la salud mental y el bienestar emocional en el adulto mayor, el personal sanitario y los propios ancianos no reconocen los problemas de salud mental en su verdadera dimensión, y es frecuente que pasen por alto los síntomas depresivos y trastornos del ánimo porque coinciden con otros problemas de salud físicos/orgánicos ${ }^{6}$. Otros autores corroboran la gran dificultad que presentan los profesionales sanitarios en reconocer y detectar las necesidades psicológicas, emocionales y espirituales de los pacientes. La carencia de herramientas, la desinformación de la repercusión en la salud, la gran dificultad para adentrarse en el interior de las personas y el paradigma biomédico que guía la atención a la salud son los principales motivos ${ }^{19}$.

\section{Concepto de serenidad, bienestar psíquico y emocional}

La Real Academia Española define serenidad como calidad de sereno. Es el valor de mantener la calma en medio de la dificultad. Si vinculamos serenidad con promoción del bienestar psíquico y emocional, podemos entenderla como un estado psicológico apacible y sosegado, relacionado con la capacidad de mantener la calma en situaciones estresantes.

El concepto de serenidad se ha definido en la literatura como un estado espiritual que disminuye el estrés y promueve una salud óptima en la persona ${ }^{20}$, un estado que, mantenido, proporciona paz interior ${ }^{21}$; también es una experiencia universal en salud relacionada con la calidad de vida ${ }^{22}$. Otros autores como Boyd-Wilson et al. ${ }^{23}$ la describen como cualidad espiritual que involucra la paz interior a pesar de las vicisitudes e incluso sentimientos, por lo que una persona puede sentir pena, pero estar serena.

El estudio de Roberts y Fitzgerald ${ }^{24}$ aporta un análisis al concepto de serenidad, implica: refugio interior de paz y seguridad; desprendimiento de excesos, deseos y emociones; y aceptación de situaciones 
Tabla 3. Revisión de subtemas

\begin{tabular}{|c|c|c|c|}
\hline \multirow{2}{*}{\multicolumn{2}{|c|}{ Temas y subtemas }} & \multicolumn{2}{|c|}{ Autores y años } \\
\hline & & 1993-2005 & 2006-2017 \\
\hline \multirow{14}{*}{$\begin{array}{l}\text { Apoyo } \\
\text { emocional en } \\
\text { la cronicidad }\end{array}$} & Investigación sobre el envejecimiento & & $\begin{array}{l}\text { Fundación general } \\
\text { CSIG }\end{array}$ \\
\hline & Predictors of adjustment to nursing home life of elderly residents & & Lee GE \\
\hline & Care of chronic patient in a complex situation: the challenge of building an integrated care & & Contel JC, Muntané B \\
\hline & Manejo del estrés en la atención a la cronicidad y la dependencia funcional & & Rodríguez Díaz MT \\
\hline & El bienestar psicológico, un indicador positivo de la salud mental & & Oramas A, Santana S \\
\hline & $\begin{array}{l}\text { Disability and poor Quality of life Associated with comorbid anxiety disorders and } \\
\text { physical condictions }\end{array}$ & & Sareen J et al \\
\hline & La salud mental y los adultos mayores & & OMS \\
\hline & Estrategia para el abordaje de la cronicidad en el Sistema Nacional de Salud & & $\begin{array}{l}\text { Sistema Nacional } \\
\text { de Salud }\end{array}$ \\
\hline & Long-term conditions and mental health, the cost of comorbidities & & $\begin{array}{l}\text { The King' fund and } \\
\text { Centre of Mental } \\
\text { Health }\end{array}$ \\
\hline & Cuidados paliativos y el apoyo emocional & & $\begin{array}{l}\text { Sociedad Española de } \\
\text { Cuidados Paliativos }\end{array}$ \\
\hline & Las enfermedades crónicas y la salud mental & & $\begin{array}{l}\text { National Institute of } \\
\text { Mental Health }\end{array}$ \\
\hline & Ansiedad y depresión en el paciente con EPOC & & Roncero C \\
\hline & Características del estado emocional en pacientes con enfermedad renal crónica & & Guadalupe et al \\
\hline & Salud mental y emociones en pacientes con enfermedades crónico-degenerativas & & González N, Tinoco A \\
\hline \multirow{8}{*}{$\begin{array}{l}\text { Concepto de } \\
\text { serenidad } \\
\text { y bienestar } \\
\text { psíquico y } \\
\text { emocional }\end{array}$} & Journal of Holistic Nursing & & Blue CL \\
\hline & Serenity for a terminally ill patient & Knioe M & \\
\hline & $\begin{array}{l}\text { The Brief Serenity Scale: A Pssychometric Analysis of a Measure of Spirituality and } \\
\text { Well-Being }\end{array}$ & & Kreitzer MJ \\
\hline & Serenity, much more than just feeling calm & & Body-Wilson BM \\
\hline & Serenity, caring with perspective & Roberts KT, Fitzgerald L & \\
\hline & Serenity as a goal for nursing practice & Whall R & \\
\hline & Serenity-Uses in the Care of Chronically ill Older patients: a concept clarification & & Norell Pejner M \\
\hline & Achieving harmony with onseself: life with a chronic illness & & Delmar $C$ et al \\
\hline \multirow{2}{*}{$\begin{array}{l}\text { Atributos y } \\
\text { niveles de } \\
\text { serenidad }\end{array}$} & Serenity, caring with perspective & Roberts KT, Fitzgerald L & \\
\hline & Serenity-Uses in the Care of Chronically ill Older patients: a concept clarification & & Norell Pejner M \\
\hline \multirow{3}{*}{$\begin{array}{l}\text { Serenidad e } \\
\text { intervención } \\
\text { enfermera }\end{array}$} & Health Promotion Practice and Its Implementation in Swedish Health Care & & Brobeck E et al \\
\hline & Serenity-Uses in the Care of Chronically ill Older patients: a concept clarification & & Norell Pejner M \\
\hline & Serenity, caring with perspective & Roberts KT, Fitzgerald L & \\
\hline
\end{tabular}


que no pueden ser cambiadas. Concluyen que la serenidad es una experiencia espiritual de paz interior que es independiente de eventos externos.

El modelo conceptual posteriormente ha sido desarrollado por Roberts y Whall25, quienes postulan que la serenidad es una emoción aprendida y positiva, que disminuye el estrés percibido y mejora la salud.

La serenidad es definida también como una experiencia emocional que contribuye a la aceptación de una situación, y se concibe como un estado de equilibrio físico, mental, emocional y espiritual que se presenta cuando una condición indeseable y desfavorable en la vida puede ser gestionada o aceptada. Experimentar la serenidad es un requisito para que el paciente sea capaz de encontrarse a sí mismo ("self") y poder continuar su vida con dignidad ${ }^{12}$.

Otros autores, como Delmar et al., afirman que es fundamental la aceptación del individuo de la nueva situación de vida causada por las enfermedades crónicas. Ello ayuda al paciente a actuar para encontrarse a sí mismo y a capacitarlo para encontrar esperanza y confianza para su futuro ${ }^{26}$.

\section{Definición de atributos y niveles de serenidad}

Una vez definido el concepto, el siguiente paso para su análisis es definir los atributos de la serenidad. Los atributos son las características conceptuales que aparecen repetidamente en la literatura especializada y puede ayudar a clarificar el concepto utilizado. Según Roberts y Fitzgerald ${ }^{24}$ se identifican diez atributos que caracterizan a las personas serenas:

1. Capacidad de estar en contacto o alcanzar un refugio interior de bienestar personal que otorga paz, seguridad y confort.

2. Capacidad de separar las emociones negativas o no deseadas y los deseos excesivos.

3. Capacidad de aceptar situaciones que no se pueden cambiar; rechazar el seguir invirtiendo energía.

4. El hábito de buscar activamente todos los caminos y medios posibles para solucionar los problemas.

5. Capacidad de vivir el presente, dejando el pasado atrás y sin preocuparse por el futuro.

6. Capacidad de perdonarse a uno mismo y a los demás; sentirse en paz con su pasado, consigo mismos y con los demás.

7. Sentido de conexión y pertinencia; se sienten conectados a alguna cosa más que a ellos mismos: Dios, naturaleza, universo, familia, grupo, animales.

8. Capacidad de entrega hacia uno mismo y hacia los demás.

9. Confianza en un poder superior; un sentido del bien sobre los acontecimientos de la vida.

10. Visión de la propia vida con una gran perspectiva, resaltando lo importante y significativo de uno mismo y de los acontecimientos vitales vividos.

En el intento de contextualizar el concepto de serenidad, diferentes autores ${ }^{11,24}$ la perciben como un estado de equilibrio para experimentar el "self" y puede estar precedida por condiciones de vida no deseables que pueden afrontarse mediante la gestión o aceptación de estas.

El "self" puede ser experimentado a través de cuatro niveles de serenidad:

- Primer nivel: es el "safe self", facilita la percepción de seguridad personal, incluyendo: capacidad de separar las emociones negativas, conexión con el refugio interior, sentido de pertenencia, confianza y resolución de problemas para escapar del peligro.
- Segundo nivel: es el "wise self"; facilita la perspectiva de la situación y se puede lograr la aceptación, incluyendo: aceptar todo aquello que no es posible cambiar, aumentar y mantener la conciencia en el presente, y cambiar todo aquello que uno mismo quiera cambiar.

- Tercer nivel: se corresponde con el "beneficent self" y establece conexión con la bondad, incluyendo: conexión con uno mismo, altruismo, elegir una postura pacífica ante la vida, perdonarse a uno mismo, a los demás y al destino.

- Cuarto y último nivel: corresponde al "universal self" y establece conexión con la espiritualidad; para alcanzar este nivel la persona tiene un sentido de conexión y pertenencia a alguna cosa más que a ellos mismos, mediante la naturaleza, el arte, la música, la meditación, logran alcanzar la espiritualidad, la conexión con la pureza del ser, la energía.

\section{Serenidad e intervención enfermera}

El impacto psicológico que genera la cronicidad está relacionado con el tipo de estrategias de afrontamiento del individuo. Los profesionales de enfermería en la atención a la cronicidad, mediante el apoyo psicológico y emocional, pueden contribuir a mejorar el afrontamiento y a disminuir los estados emocionales negativos que acompañan a la enfermedad y que retroalimentan negativamente el estado de salud del paciente $^{2}$. El apoyo emocional contempla la búsqueda de la serenidad en todos los niveles del "self", puede ayudar a los pacientes a aceptar y manejar las patologías crónicas, dando significado y dignidad a sus vidas $^{11,24}$.

A pesar de todo lo expuesto, las investigaciones sobre la serenidad corroboran que en la atención de los pacientes mayores con patologías crónicas el concepto de serenidad se utiliza en sus niveles más bajos ("safe" y "wise self") 11 . Uno de los motivos es que los profesionales de enfermería centran mayoritariamente su atención en la enfermedad del paciente y en las necesidades físicas, dejando en un segundo lugar las necesidades psicológicas, emocionales y espirituales, que asegurarían un cuidado holístico de la salud mediante la serenidad en todos los niveles del "self" 11.

Algunos estudios constatan que es necesario tener un mayor conocimiento para saber cuáles son los factores que permiten al adulto mayor con patología crónica experimentar la serenidad en todos los niveles del "self", así como estudiar cómo el profesional puede ayudar a los pacientes a encontrar la serenidad mediante intervenciones enfermeras ${ }^{11,24}$.

\section{- DISCUSIÓN}

La mayoría de los estudios encontrados sobre la serenidad están realizados en diferentes países y contextos, como Estados Unidos y Suecia. Estos ponen de manifiesto un interés especial por realizar una "exploración" del concepto de serenidad en el soporte emocional a la persona mayor con patologías crónicas. A pesar de ello, los estudios publicados constatan el poco conocimiento que hay, en general, sobre la serenidad en la práctica enfermera y su vinculación con el bienestar psíquico y emocional del adulto mayor.

Aunque mayoritariamente encontramos que la serenidad es descrita como un estado psicológico, un valor, una actitud, una experiencia, autores como Roberts y Whall indican que la serenidad es una emoción positiva, que por tanto puede ser aprendida, y que puede disminuir el estrés y mejorar la situación de salud y la calidad 
de vida de los pacientes en situación de cronicidad. De este modo, se justificaría el apoyo emocional mediante la búsqueda de la serenidad como intervención enfermera. También, se evidencia en la literatura especializada que el concepto ha de ser mejor definido, mejor explorado: clarificando y acotando el concepto, definiendo los atributos de la persona serena, validando escalas de valoración que midan la serenidad, describiendo intervenciones enfermeras que identifican cómo alcanzar la serenidad, obteniendo resultados de la efectividad de las intervenciones en la calidad de vida de los pacientes, etc.

Si algo queda claro en las investigaciones es la gran dificultad que presentan los profesionales sanitarios para reconocer y detectar las necesidades psicológicas, emocionales y espirituales de los pacientes ancianos con patologías crónicas, siendo el paradigma biomédico, el modelo asistencial que guía mayoritariamente las intervenciones del profesional en el adulto mayor con problemas crónicos.

Finalmente, resulta necesaria una mayor evidencia científica sobre los conceptos que influyen en el apoyo emocional y los beneficios obtenidos en la atención integral al adulto con problemas crónicos, que permita dar respuesta a las necesidades de salud psíquicas, emocionales y espirituales en la cronicidad y envejecimiento.

\section{- CONCLUSIONES}

Ante el avance de las enfermedades crónicas en las personas mayores resulta necesario un cambio de modelo asistencial, donde la persona, su entorno y su salud física, psicológica y emocional sean el foco central del individuo en detrimento de la mera atención a la enfermedad del modelo biomédico. El concepto de serenidad puede representar una herramienta que fomente el bienestar psíquico y emocional del paciente mayor crónico, ayudando a aceptar y a manejar la situación de su salud, así como facilitar el desarrollo de la conciencia de uno mismo y de la propia existencia ("self") para dar sentido y dignidad a la vida. Resulta imprescindible una mayor evidencia científica sobre la serenidad en la intervención enfermera, en la atención al adulto con problemas crónicos. Las enfermeras desempeñamos actividades que abarcan el cuidado emocional con serenidad. Sin embargo, es necesario que este tipo de atención sea interpretado por base científica con buena evidencia, por lo que sugerimos que el profesional ha de dar a conocer la importancia del soporte emocional en el cuidado al paciente y ha de formarse para promover las bases del saber hacer, ser y estar en el cuidado integral al individuo

\section{Conflicto de intereses}

Las autoras declaran no tener ningún conflicto de intereses.

\section{- BIBLIOGRAFÍA}

1. Contel JC, Muntané B, Camp L. Care of the chronic patient in a complex situation: the challenge of building an integrated care. Aten Primaria [Internet]. 2012 [citado 4 de enero de 2017];44(2):107-13 Disponible en: https://www.ncbi.nlm.nih.gov/pubmed/21636176

2. Rodríguez MT. Manejo del estrés en la atención a la cronicidad y la dependencia funcional. Rev electrónica geriatricarea. [Internet]. 2018 [citado 15 de enero de 2017]. Disponible en: http://geriatricarea.com/manejo-del-estres-en-la-atencion-a-la-cronicidad-y-la-dependencia-funcional/

3. Álvarez E, Almenar C, Regalado P. Estado de ánimo y depresión en las personas mayores. Fundació Salut i Envelliment UAB [Internet]. 2015 [citado 20 de febrero de 2017]. Disponible:http:// www.envellimentsaludable.com/wpcontent/uploads/2015/09/ depresion_esp.pdf

4. Cardozo IV, Rondón JE. La salud desde una perspectiva psicológica. Revista electrónica de Psicología Iztacala [Internet]. 2014 [citado 20 de febrero de 2017]: 17(3):1079-107. Disponible: http://apps.who. int/iris/bitstream/10665/186466/1/9789240694873_spa.pdf

5. Sareen J, Jacobi F, Cox BJ, Belik SL, Clara I, Stein MB. Disability and poor Quality of Life Associated with Comorbid Anxiety Disorders and Physical Conditions. Arch Intern Med [Intenet]. 2006 [citado 3 de marzo de 2017];23;166(19):2109-16. Disponible: https://www. ncbi.nlm.nih.gov/pubmed/17060541

6. OMS. La salud mental y los adultos mayores [Internet]. 2016 [citado 20 de marzo de 2017]. Disponible en: https://www.who.int/ es/news-room/fact-sheets/detail/la-salud-mental-y-los-adultos mayores

7. Ministerio de Sanidad, Servicios Sociales e Igualdad. Estrategia para el abordaje de la cronicidad en el Sistema Nacional de Salud [Internet]. 2012 [citado 22 de marzo de 2017]. Disponible: http:// webcache googleusercontent.com/search?q=cache:Vaii-Jv31YAJ:www.mscbs.gob.es/organizacion/sns/planCalidadSNS/pdf/Evaluacion_E._Cronicidad_Final.pdf+\&cd=1\&hl=es\&ct=clnk\&gl=es

8. Naylor C, Parsonage M, McDavid D, Knapp M, Fossey M, Galea A Long-term conditions and mental health, the cost of comorbidities. The King's fund and Centre of Mental Health [Internet]. 2012 [citado 4 de abril de 2017]. Disponible en: https://www.kingsfund.org.uk/ sites/default/files/field/field_publication_file/long-term-conditions-mental-health-cost-comorbidities-naylor-feb12.pdf
9. OMS. Salud mental: un estado de bienestar. [Internet]. 2013 [citado 4 de abril de 2017]. Disponible en: https://www.who.int/features/ factfiles/mental health/es/

10. Norell Pejner M, Ziegert K, Kihlgren A. Trying to cope with everyday life-Emotional support in municipal elderly care setting. Int $J$ Qual Stud Health Well-being [Internet]. 2012 [citado 4 de abril de 20171;7:10.30402. Disponible en: https://www.ncbi.nlm.nih.gov/ pmc/articles/PMC3522874/

11. Colleen D. The Spirituality Scale: Development and Psychometric Testing of a Holistic Instrument to Assess the Human Spiritual Dimension. Journal of Holistic Nursing [Internet]. 2005 [citado 15 de abril de 2017];(23):2145-67. Disponible en: https://journals.sagepub.com/doi/abs/10.1177/0898010105276180?journalCode=jhna

12. Norell Pejner M. Serenity — Uses in the Care of Chronically III Olde Patients: A Concept Clarification. Open Journal of Nursing. [Internet]. [citado 20 de marzo de 2017];5:1-9. Disponible en: https://file. scirp.org/pdf/OJN_2015011216240177.pdf

13. Caro Gutiérrez MA, Rodríguez Ríos MA, Calero C, Piattini M. Análisis y revisión de la literatura en el contexto de provectos de fin de carrera: una propuesta. [Internet]. 2010 [citado el 2 de abri de 2017]. Disponible en: https://www.researchgate.net/publication/251671565_Analisis_y_revision_de_la_literatura_en_el_ contexto de proyectos de fin de carrera Una propuesta

14. Gonzalez N, Tinocco AM, Benhumea L. Salud mental y emociones en pacientes con enfermedades crónico-degenerativas. Espacios Públicos [Internet]. 2011 [citado 22 de marzo de 2017]. Disponible en : http://www. Redalcyc.org/articulo.oa?i $d=67621319013>$ ISSN1665-8140

15. Sarró M, Ferrer-Feliu A, Rando-Matos Y, Formiga F, Rojas-Farreras S. Depresión en ancianos: prevalencia y factores asociados. Se mergen [Internet]. 2013 [citado 10 de abril de 2017];39(7):354-60. Disponible en: http://www.elsevier.es/es-revista-semergen-medicina-familia-40-articulo-depresion-ancianos-prevalencia-factores-asociados-S1138359313000178

16. Calle M, Rodriguez JL, Gómez MJ. Ansiedad y EPOC. Monog Arch Bronconeumol [Internet]. 2009:45(54):51-53. Disponible en: https://www.archbronconeumol.org/es-ansiedad-epoc-articulo-S0300289609728650
17. Norell M, Ziegert K, Kihlgren A. Dealing with daily emotions-supportive activities for the elderly in a municipal care setting. Int $J$ Qual Stud Health Well-being [Internet]. 2012 [citado 15 de abril de 2017];7:10. Disponible en: https://www.ncbi.nlm.nih.gov/pmc/articles/PMC3272264/

18. González Y, Herrera LF, López J, Nieves Z. Características del estado emocional en pacientes con enfermedad renal crónica. Rev Psicología Científica [Internet]. 2011 [citado 19 de abril de 2017]; Disponible en: http://www.psicologiacientifica.com/enfermedad-renal-cronica-pacientes-estado-emocional/

19. Muñoz A, Morales I, Berejo JC, Galán JM. La enfermería y los cuidados del sufrimiento espiritual. Index Enferm [Internet]. 2014 [citado 10 de abril de 2017]; vol. 23 n. ${ }^{0} 3$. Disponible en: http://scielo.isciii. es/scielo.php?script=sci_arttext\&pid=\$1132-12962014000200008

20. Blue $\mathrm{Cl}$. Commentary on "the brief serenity scale: a psychometric analysis of a measure of spirituality and well-being". J Holistic Nurs. 2009;27(1).17-8.

21. Knipe M. Serenity for a terminally ill patient. AJN [Internet] 1966 [citado 2 de junio de 2017];1966;66:2252-54. Disponible en: https://journals.Iww.com/ajnonline/Abstract/1966/10000/Serenity_for_a_Terminally_III_Patient.48.aspx

22. Kreitzer M. The Brief Serenity Scale: A Psychometric Analysis of a Measure of Spirituality and Well-Being. J Holist Nurs [Internet]. 2009 [citado 17 de julio de 2017];27(1);7-16. Disponible en: https:// www.ncbi.nlm.nih.gov/pmc/articles/PMC2818779/

23. Boyd-Wilson BM, Walkey FH, McClure J. Serenity, Much more than just feeling calm. Adv Psych Research [Internet]. 2004 [citado 14 de junio de 2017;2004;29:35-55. Disponible en: https://www.ncbi.nlm. nih.gov/pmc/articles/PMC2818779/

24. Roberts KT, Fitzgerald L. Serenity: caring with perspective. Sch Ing Nurs Pract. [Internet]. 1991 [citado 20 de abril de 2017];5(2):127-41. Disponible en: https://www.ncbi.nlm.nih.gov/pubmed/1891666

25. Roberts K, Whall A. Serenity as a goal for nursing practice. Image J Nurs Sch [Internet]. 1996 [citado 5 de julio de 2017]; Image: 28(4):35964. Disponible en: https://www.ncbi.nlm.nih.gov/pubmed/8987285

26. Delmar C, Boje T, Dylmer D, Forup L, Jakobsen C, Moller M, et al. Achieving harmony with onseself: life with a chronic illness. Scand J Caring Sci [Internet]. 2005 [citado 23 abril de 2017];19(3):204-12. Disponible en: https://www.ncbi.nlm.nih.gov/pubmed/16101848 